\title{
Review on the Analysis Methods of Starch, Amylose, Amylopectinin Food and Agricultural Products
}

\author{
Edy Subroto, Georgina Jeanette, Yunita Meiyanasari, Ikming Luwinsky, Savix Baraddiaz \\ Department of Food Industrial Technology, Faculty of Agro-Industrial Technology, UniversitasPadjadjaran, \\ Jl.Raya Bandung-Sumedang Km. 21, Jatinangor, Sumedang 40600, Indonesia. Email:edy.subroto@ unpad.ac.id
}

\begin{abstract}
Starch is one of the biggest components of carbohydrates in food and other biological materials. The main components of starch consist of amylose and amylopectin. The comparison between amylose and amylopectin greatly influences the functional, physicochemical, and pasting properties of starch. Various methods of analyzing starch, amylose, and amylopectin content have been widely studied for various purposes. This review describes several types of quantitative analysis methods for starch, amylose and amylopectin. Quantitative methods to determine starch content include the polarimetry, anthrone, Fourier-transform infrared spectroscopy (FTIR), spectrophotometry, penetrometry, and Luff schoorl. The methods for determining amylose content are spectrophotometry and gravimetry, while the method for determining amylopectin content using X-Ray Diffraction (XRD) and gravimetry. Each method has several advantages and disadvantages so that it can be used to determine the method suitable for materials with certain characteristics.
\end{abstract}

Key words: Starch, amylose, amylopectin, analysis method.

\section{INTRODUCTION}

The source of carbohydrates in food mostly comes from starch which is a reserve polysaccharide in plants in the form of water-insoluble crystallized granules [1]. Agricultural products that contain a lot of starch and have been widely used by industry include starch from cassava, sweet potatoes, sago, rice, corn, bananas, and potatoes. Technological developments have made starch modified to obtain the desired characteristics [2]-[4]. The main components of starch comprise amylose and amylopectin. The content of amylose and amylopectin in starch is affected by the type, age, and condition at the time of harvest [5]. The quality of foodstuffs that contain starch is very much affected by the levels of amylose and amylopectin. This is due to the role of amylose and amylopectin in the process of gelatinization, retrogradation and functional characteristics of starch [6].

Various analyzes have been developed to determine the starch content, amylose content and amylopectin in food through various methods including physical, chemical and biotechnological. Physical and chemical methods are relatively easier to do, whereas biotechnological methods are more difficult to do, but the results are very specific and promising as biotechnology develops for various purposes of analysis and production processes [7], [8]. In the starch analysis, starch granules are separated from the main components by grinding, sedimentation in the water, filtering and centrifugation, washing, and drying. Starch granules cannot dissolve in water and have a high density so that if centrifugation is carried out, the starch will easily settle to the bottom, which is then easily separated. In foodstuffs that have undergone processing, starch analysis requires a drying process first, precipitating and then dissolving again in an $80 \%$ ethanol solution that has been heated.

Starch content in food can be identified by the polarimetry, anthrone, Fourier-transform infrared spectroscopy (FTIR), spectrophotometry, penetrometry, and Luff schoorl methods. Amylose content in starch can then be determined by spectrophotometric analysis and gravimetry. Separation or isolation of amylose and amylopectin can be conducted by separation based on its solubility to obtain amylose and amylopectin with good purity [9]. Determination of amylopectin levels can use several analytical methods, including the method of X-Ray Diffraction (XRD), spectrophotometry, and gravimetry. Amylose and amylopectin in starch can also be analyzed qualitatively by iodine. Amylose-containing samples produce a blue color with iodine by heating with dilute acid, while a positive sample of amylopectin produces a red color [10]. This review discusses several methods of analyzing starch, amylose and amylopectin content that have been used by researchers.

\section{ANALYSIS METHODS OF STARCH}

\subsection{Starch analysis by polarimetry}

Starch content can be measured by the polarimetry method. This method includes a fast measurement method for starch and sugar content. The instrument used for this method is a polarimeter. This method has an accuracy rate of around $0.5 \%$ [11]. The polarimeter is a scientific instrument used to measure the cause of the rotation angle by using continuous polarization light on the active optical substance. Each type of sugar or carbohydrate has a different rotation angle, including 
starch [12]. The polarimeter is often used for various starch and other carbohydrate analyzes in the fields of pharmaceuticals, food and beverages, sugar, and cosmetics. The role of the analyzer is the polaroid which analyzes or polarizes the light.

The principle of the polarimetry method is the characteristics of carbohydrates that can rotate polarized light fields to the right (+) and to the left (-). Polarimeter uses optical activity measurements before and after dextran hydrolysis by the enzyme of dextranase. The sample solution is then inserted into a polariscope tube of a certain length. The factor seen in this measurement is the angle of rotation produced by the bending of the light due to polarization. The sample preparation needs to be conducted so that the results obtained have good accuracy. The sample to be observed is first put into a boiling calcium chloride solution and heated for 15 minutes. Calcium chloride solution must have a $\mathrm{pH}$ of 2.5 and a specific gravity of 1.3. The control used at this stage is n-octyl alcohol. The volume of the solution during the boiling process is controlled by adding water [11].

\subsection{Starch analysis by Anthrone method}

The anthrone method is one of the colorimetric methods used to determine starch concentration or total sugar content in food. Under acidic conditions, sugars react with anthrone reagents to produce a blue-greenish color. Heating needs to be conducted when reacting samples with sulfuric acid and reagents with the aim of accelerating the reaction rate, then cooled and measured its absorbance at $630 \mathrm{~nm} \mathrm{[13].} \mathrm{The}$ anthrone method can determine the presence of reducing sugars and non reducing sugars because they are reacted with sulfuric acid as a strong oxidizing agent. Nonetheless, research conducted by Alkayyis and Susanti [13] shows that the anthrone method is less accurate in determining starch content because of the low sensitivity when tested for sensitivity.

\subsection{Starch analysis by Fourier transform infrared spectroscopy (FTIR) method}

FTIR is an easy method of analyzing starch content because this technique does not require complicated sample preparation and does not require special expertise to master this tool [14]. The principle of FTIR is the characteristic of the bond and the composition in the sample will affect the emission of light monochromatic light in energy absorption which will reflect the quantity detected by the instrument and used to measure the quantity of infrared energy absorbed. The spectrum collected in the infrared region is represented graphically as the energy absorbed as a function of wavelength. FTIR is very practical and suitable for the determination of organic compounds from biological substances, including starches and other carbohydrates [15].

In general, the steps in determining starch content by FTIR are using the ATR-Total Reflectance Attenuation technique; then the sample is placed in the sample area with the air spectrum used as a background. Then the spectrum was recorded using Pro Variant Resolution, and starch samples were measured in the region of 4000-700 cm-1; each spectrum was measured 40 times [14]. In recent years, this technology or method is widely used in various agribusiness industries, the food industry [15], and the pharmaceutical industry [16]. FTIR is preferred mainly because of its rapid analysis, high precision, easy and simple handling, low cost per sample, easy to obtain reference methods, and no or little sample preparation [17].

\subsection{Starch analysis by Spectrophotometry}

Ultraviolet-visible (UV-VIS) spectrophotometry method is a method with the principle of color formation in analytes with reagents used so that it can absorb light at specific wavelengths specifically. The use of color-forming reagents will increase sensitivity, so the detection limit is low. Several studies that have succeeded in measuring amylose in analog rice based on flour and purple sweet potato starch. The principle of this method is to prove that in the validation of the analysis method there are several parameters that must be considered including accuracy, precision, the limit of detection (LOD), the limit of quantitation (LOQ), and linearity [18].

\subsection{Starch analysis by Penetrometry}

The penetrometry method is one alternative that can be developed to predict starch content with the dry method. The analysis was carried out by measuring the hardness of the material using a penetrometer. This method has long been used to measure the hardness of various agricultural commodities containing starch, such as potatoes, sweet potatoes, cassava, and others. The principle of the analysis with a penetrometer is that the penetrometer rod is held and the penetrometer needle is carefully pricked vertically on the surface. This is more practical, does not require a lot of money, is easy to use, easy to carry and store [19].

\subsection{Starch analysis by Luff Schoorl Method}

Luff Schoorl method is a method that is often used to determine starch content and is a good method for measuring carbohydrate levels with an error of $\pm 10 \%$. The principle used is the iodometric reaction with the process of titration of free iodine (I2) in solution, then the starch content can be seen from the level, amount, and shape of the starch [20].

\section{ANALYSIS METHODS OF AMYLOSE}

Amylose is a soluble part of the starch. Amylose has a straight-chain polymer structure formed by an arrangement of about $1000 \alpha$-glucose molecular units bound by $\alpha$ $(1,4)$-glycosidic bonds to form a straight-chain polymer [21], [22]. Amylose has a solid helical structure; therefore, amylose is more resistant to digestion than other starch molecules, 
which is an important characteristic of resistant starch. Strong molecular interactions are formed because of the simple structure of the amylose helical interaction that occurs in amylose molecular groups. A simpler structure of amylose makes hydrogen bonds between amylose molecules also easier than amylopectin. Amylose has fewer branches compared to amylopectin, which makes it more resistant to digestion because amylose forms crystals more easily [23]. In most foodstuffs, amylose is a component of starch which amounts to around 20-30\% [24].

Amylose plays an important role in storing energy in plants. Amylose is more difficult to digest than amylopectin, but amylose has a compact structure, so it only requires less space than amylopectin. Therefore, amylose is the preferred starch for storage in factories [24]. Amylose can act as an emulsion stabilizer, water binder, thickener, and gel-forming agent in the food industry. The helical amylose chain has a hydrophobic part that can bind the hydrophobic molecules such as aromatic compounds and lipids to form complex compounds. But there is a problem that when crystallizing or associating, it can lose stability, namely the ease of releasing water in processing and storage, known as syneresis. Amylose gel adhesiveness decreases when amylose concentration increases, but the integrity of the gel increases. The viscosity can be affected when other things, including amylopectin bind to amylose, but by adding xanthan gum, alginate, $\kappa$-carrageenan, or low molecular weight sugars can increase stability against syneresis. The ability of amylose to bind water can improve food quality and can be a substitute for fat [23].

Amylose content in starch greatly affects the texture and characteristics of the materials containing the starch. These characteristics are important to know to study the processes that occur within them, such as retrogradation and gelatinization. Amylose content can be analyzed quantitatively. Some quantitative methods of amylopectin analysis include gravimetry and spectrophotometry.

\subsection{Amylose analysis by gravimetry}

Amylose content in agricultural products can be determined by measuring the weight of components in a pure state after going through a process of separation or isolation. The amylose separation process is carried out by means of a starch solution heated at a constant temperature of $100{ }^{\circ} \mathrm{C}$ to form a gel. The gel was filtered using filter paper then the filtrate obtained was added to a phosphate buffer to a $\mathrm{pH}$ of around 6.3. The solution was reheated for 2 hours at $100{ }^{\circ} \mathrm{C}$. After that, a complex solution of $\mathrm{n}$-Butanol was added, and the solution was reheated for 1 hour at $100{ }^{\circ} \mathrm{C}$, then cooled at room temperature for 24 hours to form a precipitate which is an amylose fraction. The residue was added with methanol to form a white precipitate and then filtered again until a pure white precipitate was obtained.

In this gravimetric method, there are three variables that affect the amylose content of the sample, namely $\mathrm{pH}$, the amylose separation or isolation process, and the ratio between complex solutions and starch solutions. The best conditions for the analysis of amylose gravimetric methods are at $\mathrm{pH} 6.5$, the isolation of amylose by the salting-out method, and the ratio of complex solutions to starch solutions are 2: $3.9(\mathrm{v} / \mathrm{v})$ [9]. The advantages of this method are its easy preparation and a high level of accuracy. But the gravimetric method is not recommended for long-term field measurements, but this method is good if conducted at a laboratory scale.

\subsection{Amylose analysis by Spectrophotometry}

Amylose content in starch from food materials can be determined based on the interaction between amylose and light at certain wavelengths, which is known as the spectrophotometric method. Determination of amylose content by this method can be started by the preparation of the material that is extracting starch from the sample by immersing the sample, then smoothing and filtering [25]. The filtrate obtained is then allowed to stand until sludge is formed and then the sludge is washed with distilled water to obtain a white precipitate which is starch, then the amylose and amylopectin were separated by heating at $50{ }^{\circ} \mathrm{C}$ for 30 minutes.

Heating white precipitates (starch) to separate amylose and amylopectin can occur due to the presence of components that are easily soluble in hot water namely amylose and components that are insoluble in hot water (amylopectin) [26]. The heated starch was then added trichloroacetic acid and 50 $\mu \mathrm{L}$ of iodine solution (Tortoe et al., 2017). The solution will turn blue. Amylose content is then calculated based on the blue color intensity of the solution measured by a spectrophotometer at $625 \mathrm{~nm}$. The amylose content can be determined based on the standard curve equation of amylose.

The requirement for the absorbance of the sample to be measured by the spectrophotometric method is that the compound must have a chromophore group. The starch solution does not have the chromophore group; therefore, reactants need to be added, which can make the starch solution have a colored absorption spectrum. Iodine solution provides a solution containing amylose in blue. This is because the iodine solution can bind to amylose because of its straight-chain structure [10].

\section{ANALYSIS METHODS OF AMYLOPECTIN}

Amylopectin is a type of carbohydrate molecule that has many branches with $\alpha$-1,4-glycosidic in linear and $\alpha-1,6$-glycosidic in branching chains. Amylopectin molecule has 1 type of chain, namely $\mathrm{C}$ chain (backbone) with one end consisting of reducing sugar groups and the other end in the form of connecting branches of another amylopectin (chains A and B) [28]. Chain B can link directly with chain C, while chain $A$ is a chain that links with chain B [29]. The amylopectin chain that joins plays a role in forming the crystal structure of starch [30], so that the more amylopectin components in starch, the texture becomes glassy or waxy. An amylopectin molecule can be composed of 1000 glucose units. Amylopectin is the largest component in most starches 
of agricultural products, and the amount can reach $70-85 \%$ [31]. Amylopectin content in starch greatly affects the texture and nature or characteristics of the starch. These characteristics are important to know to study the processes that occur within them, such as retrogradation and gelatinization. Some quantitative methods of amylopectin analysis include X-Ray Diffraction, spectrophotometry, and gravimetry.

\subsection{Amylopectin analysis by X-Ray Diffraction (XRD) method}

XRD is a method that can be used to identify the crystal structure of the observed sample [32], in this case, in the form of amylopectin. Its working principle is that it diffracts a beam of X-rays in all directions until the structure of the atoms and molecules of a crystal is identified. In this method, the angle and intensity of the X-ray diffracted can be measured to produce a three-dimensional picture of the electron density in the crystal [33]. This method is also called X-Ray Crystallography. The XRD method can provide detailed information about the crystal structure of the observed sample, its chemical composition, and its physical components [34]. This method has a high accuracy.

The tool commonly used in the XRD method to identify amylopectin crystals is the Wide Angle X-Ray Diffractometer (WAXS). The principle of this method is to measure the reflector angle of the sample. The angle formed is in the range $4-33^{\circ}(2 \theta)$ with a capture time of 2 seconds per step. The diffractometer is equipped with a detector of silicon. The anode uses Co K $\alpha 1$ radiation with a wavelength of $1.7902 \AA$ and is made monochrome with a Ni filter [29].

Before testing by the XRD method, it is necessary to prepare the sample. Samples to be observed, the content and structure of amylopectin are first separated from other components. Separation can be conducted by soaking and heating at $80{ }^{\circ} \mathrm{C}$ for 2 hours [35]. The separated product is then dried in an oven to form amylopectin powder. This powder can then be used as an observation sample on WAXS. The proportion of the crystal content is known by reducing the background value to the value obtained from the WAXS sample [29].

\subsection{Amylopectin analysis by Spectrophotometry}

Spectrophotometry is a method for measuring the quantity of a sample by absorbing or transmitting wavelengths of the sample to its interaction with UV light, visible light, and infrared [36]. The spectrophotometric method uses a spectrophotometer, generally a UV-Visible type. The energy produced by UV light, visible light, and infrared in the range of 1-5 eV can pass through bonds formed between atoms of molecules and crystals with a very broad type. Spectrophotometry can identify the type and the content of molecules, and the physical and chemical properties of the sample through the excitation energy it produces [37].

Samples to be analyzed by spectrophotometric method need to be prepared by extracting starch and separated between amylose and amylopectin first. According to Ma and Deng [38], separation can be conducted by heating at $100{ }^{\circ} \mathrm{C}$ for 1 hour and then cooling. After the temperature drops to 55 ${ }^{\circ} \mathrm{C}$, the $\mathrm{pH}$ of the solution is adjusted to 6.3 using phosphate buffer to be heated again at $121{ }^{\circ} \mathrm{C}$ for 3 hours. The next process is heating with a hot plate at a temperature of $100{ }^{\circ} \mathrm{C}$. $\mathrm{N}$-butanol is added to the solution at a ratio of $1: 3$ and stirred until the temperature drops again to $55^{\circ} \mathrm{C}$. The solution is then allowed to stand overnight and centrifuged to separate the amylose fraction, amylopectin, and other impurities. Amylopectin extract is then dried and ground to a powder [38]. It aims to obtain accurate results when analyzed with a spectrophotometer. In addition to heating, preparation can also be conducted by the cold alkaline method. Determination with a spectrophotometer requires a standard curve, and the wavelength used is $620 \mathrm{~nm}$ [39].Spectrophotometry is an analytical instrument that is widely used and continues to grow with various modifications in its application, including through Algorithm-based Spectrophotometry[40].The data obtained can be further processed or analyzed for a variety of more communicative data displays, including by the regression testing for test effectiveness technique[41].

\subsection{Amylopectin analysis by gravimetry}

Gravimetric method is a quantitative method that can measure the content of an analyte based on its solid mass. This method requires sample extracts with good quality and purity because impurities that are weighted can affect the yield and measurement accuracy [42]. The gravimetric method begins with the separation between amylose and amylopectin. This separation can be conducted by heating [26] or by complexing compounds in the form of butanol [9]. The starch that has separated between amylose and amylopectin is then dried in an oven at $70{ }^{\circ} \mathrm{C}$ to a constant weight. The constant weighing is the weight of starch. Amylopectin content is the result of the subtraction in amylose content [43].

\section{CONCLUSION}

Starch is composed of amylose and amylopectin. Methods that can be used to analyze the starch content of an agricultural product include the methods of polarimetry, anthrone, FTIR, spectrophotometry, penetrometry, and Luff schoorl. Amylose content of a starchy material can be analyzed by using the spectrophotometry and gravimetry. While the methods that can be used to analyze the amylopectin content of a material, including the X-Ray diffraction (XRD), spectrophotometry, and Gravimetry.

\section{ACKNOWLEDGEMENT}

The authors would like to thank the Rector of UniversitasPadjadjaran and the Ministry of Education and Culture of the Republic of Indonesia for the support provided. 


\section{REFERENCES}

1. A. Lovegrove, C. H. Edwards, I. De Noni, H. Patel, S. N. El, T. Grassby, C. Zielke, M. Ulmius, L. Nilsson, P. J. Butterworth, P. R. Ellis, and P. R. Shewry, Role of polysaccharides in food, digestion, and health, Crit. Rev. Food Sci. Nutr., vol. 57, no. 2, pp. 237-253, Jan. 2017.https://doi.org/10.1080/10408398.2014.939263

2. T. Adiyanti and E. Subroto, Modifications Of Banana Starch And Its Characteristics : A Review, Int. J. Sci. Technol. Res., vol. 9, no. 3, pp. 3-6, 2020.

3. S. C. Alcázar-Alay and M. A. A. Meireles, Physicochemical properties, modifications and applications of starches from different botanical sources, Food Sci. Technol., vol. 35, no. 2, pp. 215-236, 2015.https://doi.org/10.1590/1678-457X.6749

4. E. Subroto, R. Indiarto, H. Marta, and S. Shalihah, Effect of heat - moisture treatment on functional and pasting properties of potato (Solanumtuberosum $L$. var. Granola) starch, Food Res., vol. 3, no. October, pp. 469-476, 2019.https://doi.org/10.26656/fr.2017.3(5).110

5. A. L. Charles, Y. H. Chang, W. C. Ko, K. Sriroth, and T. C. Huang, Influence of Amylopectin Structure and Amylose Content on the Gelling Properties of Five Cultivars of Cassava Starches, J. Agric. Food Chem., vol. 53, no. 7, pp. 2717-2725, Apr. 2005.https://doi.org/10.1021/jf048376+

6. J. Jane, Y. Y. Chen, L. F. Lee, A. E. McPherson, K. S. Wong, M. Radosavljevic, and T. Kasemsuwan, Effects of amylopectin branch chain length and amylose content on the gelatinization and pasting properties of starch, Cereal Chem., vol. 76, no. 5, pp. 629-637, 1999.https://doi.org/10.1094/CCHEM.1999.76.5.629

7. D. C. V. Arifin, D. I. Saragih, and S. J. Santosa, Antibacterial Activity of Silver Nanoparticles Synthesized Using Tyrosine as Capping and Reducing Agent, Int. J. Emerg. Trends Eng. Res., vol. 8, no. 6, pp. 2414-2421,

2020.https://doi.org/10.30534/ijeter/2020/34862020

8. E. Subroto and F. Hayati, Chemical and Biotechnological Methods for the Production of Xylitol : A Review, Int. J. Emerg. Trends Eng. Res., vol. 8, no. 6, pp. 2508-2512, 2020 https://doi.org/10.30534/ijeter/2020/49862020

9. A. Niken and Adepristian, IsolasiAmilosadanAmilopektindariPatiKentang, J. Tekonologi Kim. dan Ind., vol. 2, no. 3, pp. 57-62, 2013.

10. E. Bertoft, Understanding Starch Structure : Recent Progress, Agronomy, vol. 7, no. 56, pp. 1-29, 2017.https://doi.org/10.3390/agronomy7030056

11. K. A. Clendenning, Polarimetric Determination of Starch in Cereal Products: I. Rapid Determination of Starch in Crude Gluten, Can. J. Res., vol. 20, pp. 403-410, 2011.https://doi.org/10.1139/cjr42c-036

12. F. Dimins, P. Kuka, and I. Cakste, Content of carbohydrates and specific rotation angle of honey, in Foodbalt - 3rd Baltic Conference on Food Science and Technology, 2008, pp. 121-125.
13. H. K. Al-kayyis and H. Susanti, PerbandinganMetodeSomogyi-Nelson Dan Anthrone-SulfatPadaPenetapan Kadar GulaPereduksiDalamUmbiCilembu (Ipomeabatatas L.), J. Pharm. Sci. Community, vol. 13, no. 2, pp. 81-89, 2016.https://doi.org/10.24071/jpsc.2016.130206

14. A. Bartošová, M. Soldán, M. Sirotiak, L. Blinová, and A. Michaliková, Application of FTIR-ATR Spectroscopy for Determination of Glucose in Hydrolysates of Selected Starches, Res. Pap. Fac. Mater. Sci. Technol. Slovak Univ. Technol., vol. 21, pp. 116-121, 2013.https://doi.org/10.2478/rput-2013-0019

15. J. J. L. Mendoza, C. A. S. Sierra, and J. J. Morales, Evaluation of the Starch Quantification Methods of Using Factorial Experiments, Int. J. Food Sci., vol. 2018, 2018.https://doi.org/10.1155/2018/5901930

16. D. Chakraborty, Instrumentation of FTIR and Its Herbal Applications, World J Pharm Pharm. Sci, vol. 5, no. 3, pp. 498-505, 2016.

17. C. Siregar, S. Martono, and A. Rohman, Application of Fourier Transform Infrared ( FTIR ) Spectroscopy Coupled with Multivariate Calibration for Quantitative Analysis of Curcuminoid in Tablet Dosage Form, J. Appl. Pharm. Sci., vol. 8, no. 8, pp. 151-156, 2018.https://doi.org/10.7324/JAPS.2018.8821

18. M. A. Christianty, Y. Martono, and C. A. Riyanto, ValidasiMetodeAnalisisAmilosasecaraSpektrofotome tri Ultraviolet-Visible (UV-Vis) dalamSingkong, in Seminar NasionalBiologidanPendidikan UKSW, 2018.

19. S. Nurdjanah, Susilawati, and M. R. Sabatini, Prediction of cassava starch content at different stages of maturity using penetrometer, J. Teknol. dan Ind. Has. Pertan., vol. 12, no. 2, pp. 65-73, 2007.

20. Ifmaily, Penetapan Kadar PatipadaBuahManggaMuda (MengiferaIndica L) MenggunakanMetode Luff Schoorl, J. Katalisator, vol. 3, no. 2, pp. 106-113, 2018.

21. J. A. Rendleman, molecular mass, Biotechnol. Appl. Biochem., vol. 178, pp. 171-178, 2000.

22. J. Kadokawa, K. Yano, S. Orio, and K. Yamamoto, Formation of Supramolecular Soft Materials from Amylosic Inclusion Complexes with Designed Guest Polymers Obtained by Vine-Twining Polymerization, ACS Omega, vol. 4, no. 4, pp. 6331-6338, Apr. 2019.https://doi.org/10.1021/acsomega.9b00238

23. D. F. Birt, T. Boylston, S. Hendrich, J-L. Jane, J. Hollis, L. Li, J. McClelland, S. Moore, G. J. Phillips, M. Rowling, K. Schalinske, M. P. Scott, and E. M. Whitley, Resistant starch: promise for improving human health, Adv. Nutr., vol. 4, no. 6, pp. 587-601, 2013.https://doi.org/10.3945/an.113.004325

24. J. Wang, P. Hu, Z. Chen, Q. Liu, and C. Wei, Progress in High-Amylose Cereal Crops through Inactivation of Starch Branching Enzymes, Front. Plant Sci., vol. 8, no. April, $\quad$ pp. 1-10, 2017.https://doi.org/10.3389/fpls.2017.00469

25. M. Chen and C. J. Bergman, Method for determining the amylose content , molecular weights , and weightand molar-based distributions of degree of polymerization of amylose and fine-structure of 
amylopectin, Carbohydr. Polym., vol. 69, pp. 562-578, 2007.https://doi.org/10.1016/j.carbpol.2007.01.018

26. H. Pramesti, Siadi, and Cahyono, AnalisisRasio Kadar Amilosa/AmilopektindalamAmilumdariBeberapaJen isUmbi,” Indo. J. Chem. Sci., vol. 4, no. 1, 2015.

27. C. Tortoe, P. T. Akonor, K. Koch, and C. Menzel, Amylose and amylopectin molecular fractions and chain length distribution of amylopectin in 12 varieties of Ghanaian sweet potato ( Ipomoea batatas ) flours, Int. J. Food Prop., vol. 20, no. 12, pp. 3225-3233, 2017.https://doi.org/10.1080/10942912.2017.1283326

28. J. N. BeMiller, Starches: Molecular and Granular Structures and Properties, in Carbohydrate Chemistry for Food Scientists, 3rd ed., AACC International, 2019, pp. 159-189.

29. B. Martens, Gerrits, Bruininx, and Schols, Amylopectin Structure and Crytallinity Explains Variation in Digestion Kinetics of Starches Across Botanic Sources in an In Vitro Pig Model, J. Anim. Sci. Biotechnol, vol. 9, no. 91,2018 https://doi.org/10.1186/s40104-018-0303-8

30. A. Gunaratne and H. Corke, Starch: Analysis of Quality. Elsevier, 2016.

31. K. F. Tiefenbacher, Glossary in Terms of Wafers, Waffles, and Adjuncts, in The Technology of Wafers and Waffles II: Recipes, Product Development and Know-How, Academic Press, 2019, pp. 325-411.

32. A. A. Bunaciu, E. G. Udristioiu, and H. Y. Aboul-Enein, $X$-Ray Diffraction: Instrumentation and Applications, Crit. Rev. Anal. Chem., vol. 45, no. 4, pp. 289-299,

2015.https://doi.org/10.1080/10408347.2014.949616

33. L. Duta, N. Mihailescu, A. C. Popescu, C. R. Luculescu, I. N. Mihailescu, G. Çetin, O.Gunduz, F.N.Oktar, A.C.Popa, A.Kuncser, C.Besleaga, G.E.Stan, Comparative Physiscal, Chemical and Biological Assesment of SImple and Titanium-Doped Ovine Dentine-Derived Hydroxyapatite Coatings Fabricated by Pulsed Laser Deposition, Appl. Surf. Sci., vol. 413, pp. 129-139, 2017. https://doi.org/10.1016/j.apsusc.2017.04.025

34. F. Sima, C. Ristoscu, L. Duta, O. Gallet, K. Anselme, and I. N. Mihailescu, Laser Thin Films Deposition and Characterization for Biomedical Application, in Laser Surface Modification of Biomaterials: Techniques and Applications, R. Vilar, Ed. Woodhead Publishing, 2016, pp. 77-125.

https://doi.org/10.1016/B978-0-08-100883-6.00003-4

35. M. Todica, E. M. Nagy, C. Niculaescu, O. Stan, N. Cioica, and C. V. Pop, XRD Investigation of Some Thermal Degraded Starch Based Materials, J. Spectrosc., vol. 2016, pp. 1-6, 2016 https://doi.org/10.1155/2016/9605312

36. T. A. Germer, J. C. Zwinkels, and B. K. Tsai, Intoduction, in Experimental Methods in the Physical Sciences, Vol. 46, Elsevier, 2014, pp. 1-9.

37. P. C. Martin and M. B. Eyring, Microspectrophotometry, in Experimental Methods in the Physical Sciences, Vol. 46, Elsevier, 2014, pp. 489-517.
38. H.-W. Ma and M. Deng, An Optimized Procedure for Determining the Amylase/Amylopectin Ratio in Common Wheat Grains Based on the Dual Wavelength Iodine-Binding Method, J. Genet. Genet. Eng., vol. 1, no. 1, pp. 23-30, 2017.

39. S. Nakamura, H. Satoh, and K. Ohtsubo, Development of Formulae for Estimating Amylose Content, Amylopectin Chain Length Distribution, and Resistant Starch Content Based on Iodine Absorption Curve of Rice Starch, J. Biosci. Biotechonology, Biochem., vol. 79, no. 3, pp. 443-455, 2014.https://doi.org/10.1080/09168451.2014.978257

40. K. M. R. Tabal, Nitrogen deficiency level assessment device for rice (Oryza sativa L.) and maize (zea mays L.) using classification algorithm-based spectrophotometry, Int. J. Adv. Trends Comput. Sci. Eng., vol. 9, no. 3, pp. 2834-2841, 2020. https://doi.org/10.30534/ijatcse/2020/54932020

41. O. Dahiya, K. Solanki, S. Dalal, and A. Dhankhar, Regression testing: Analysis of its techniques for test effectiveness, Int. J. Adv. Trends Comput. Sci. Eng., vol. 9, no. 1, pp. 737-744, 2020. https://doi.org/10.30534/ijatcse/2020/105912020

42. D. Harvey, Gravimetric Methods, in Analytical Chemistry, DePauw University, 2016, pp. 337-389.

43. N. Palupi, Kusnandar, and Lestari, Biological Values of Dried Corn Noodles Substituted with Heat Moisture Treated (HMT)-Corn Flour, J. Teknol. dan Ind. Pangan, vol. 25, no. 2, pp. 6-16, 2015. https://doi.org/10.6066/jtip.2015.26.1.9 